VOL. 51 (1995) [291-300]

\title{
HIGHER ORDER GATEAUX SMOOTH BUMP FUNCTIONS ON BANACH SPACES
}

\author{
David P. McLaughlin and Jon D. VanderwerfF
}

\begin{abstract}
For $\Gamma$ uncountable and $p>1$ odd, it is shown $\ell_{p}(\Gamma)$ admits no continuous $p$ times Gateaux differentiable bump function. A space is shown to admit a norm with Hölder derivative on its sphere if it admits a bounded bump function with uniformly directionally Hölder derivative. Some results on smooth approximation are obtained for spaces that admit bounded uniformly Gateaux differentiable bump functions.
\end{abstract}

\section{INTRODUCTION}

Because of their intimate relationship with geometric properties, the existence of real-valued functions with bounded nonempty support of a given degree of smoothness (that is, smooth bump functions) is a widely studied topic in Banach space theory (see [3]). While the questions concerning the best possible order of Fréchet smoothness for bump functions and norms on $L_{p}$ were completely solved by Bonic and Frampton [1], the corresponding questions for Gateaux smoothness have remained largely unsolved. In this direction, Troyanski [10] has shown for $p$ odd, $L_{p}(\mu)$ admits a $p$-times Gateaux differentiable norm if $\mu$ is sigma finite, while $\ell_{p}(\Gamma)$ does not admit a $p$-times Gateaux differentiable norm if $\Gamma$ is uncountable. Fabian, Whitfield and Zizler [5] have shown that no $L_{p}$ space for $1 \leqslant p<2$ can admit a twice Gateaux differentiable norm. A dramatically simpler proof of this can be obtained using arguments of Borwein and Noll from [2, Proposition 2.2]. In fact, [8] applies the techniques of [2] to show a wide class of spaces, including $L_{p}$ for $1 \leqslant p<2$, cannot admit continuous twice Gateaux differentiable bump functions.

This note presents results which provide further evidence of the strength of higher order and uniform Gateaux differentiability. The first section improves Troyanski's result [10, Theorem 3.5] by showing if $p>1$ is odd and $\Gamma$ is uncountable, then $\ell_{p}(\Gamma)$ admits no continuous $p$-times Gateaux differentiable bump function. This is not just a formal improvement, because Haydon's striking results $[6,7]$ show there

Received 19th May, 1994

This work was undertaken whilst the second author was a NSERC postdoctoral fellow. The authors wish to thank V. Zizler for helpful discussions and suggestions concerning this note.

Copyright Clearance Centre, Inc. Serial-fee code: 0004-9729/95 \$A2.00+0.00. 
are spaces that admit Lipschitz $C^{\infty}$-smooth bump functions but have no Gateaux differentiable norms. In contrast to this, the second section shows spaces that admit Lipschitz uniformly Gateaux differentiable bump functions must have Gateaux smooth norms. More restrictively, we show a space admits a norm with Hölder derivative on its sphere if it has a Lipschitz bump function whose derivative is uniformly directionally Hölder; this sharpens [5, Theorem 3.2].

Throughout this note, $X$ and $Y$ will denote real Banach spaces. The continuous dual of $X$ is written $X^{*}$. The closed unit ball and unit sphere on $X$ will be denoted by $B_{X}$ and $S_{X}$ respectively. Because norms are not differentiable at the origin, we say a norm is, for example, Gateaux differentiable, if it is Gateaux differentiable at all nonzero points.

\section{Gateaux BUmp functions on $\ell_{p}(\Gamma)$}

We shall say the function $\phi: X \rightarrow \mathbb{R}$ is Gateaux differentiable at $x$, if there is a $\Lambda \in X^{*}$ such that $\lim _{t \rightarrow 0}[\phi(x+t h)-\phi(x)] / t=\Lambda(h)$ for each $h \in X$; as in [10] we shall say $\phi$ is twice Gateaux differentiable at $x$ if $\phi^{\prime}(v) \in X^{*}$ exists in the Gateaux sense for each $v$ in a neighbourhood of $x$ and

$$
\phi^{\prime \prime}(x)(h, k)=\lim _{t \downarrow 0} \frac{1}{t}\left(\phi^{\prime}(x+t h)-\phi^{\prime}(x)\right)(k)
$$

exists and is a continuous symmetric bilinear form. Higher order Gateaux differentiability can be defined inductively.

A collection $\left\{x_{\gamma}, f_{\gamma}\right\} \subset X \times X^{*}$ is called a Markuševič basis of $X$ if $f_{\gamma}\left(x_{\alpha}\right)=1$ for $\alpha=\gamma$ and 0 otherwise, $\overline{\operatorname{span}}\left(\left\{x_{\gamma}\right\}_{\gamma}\right)=X$, and $\left\{f_{\gamma}\right\}_{\gamma}$ is total on $X$ (that is, $f_{\gamma}(x)=0$ for all $\gamma$ if and only if $x=0$ ).

Lemma 1.1. Suppose $X$ is reflexive, $\left\{x_{\gamma}, f_{\gamma}\right\}_{\gamma}$ is an $M$-basis of $X$ with $\left\|x_{\gamma}\right\| \leqslant$ 1 , and $\phi: X \rightarrow \mathbb{R}$ is weakly sequentially continuous with $\phi(0)=0$. Then $\left\{\gamma: \phi\left(x_{\gamma}\right) \neq\right.$ $0\}$ is countable.

Proof: Following [10, Lemma 3.7], consider $\left\{x_{\gamma}:\left|\phi\left(x_{\gamma}\right)\right| \geqslant \varepsilon\right\}$. If this set is infinite, by its relative weak compactness and the Eberlein-Smulyan theorem it has a weakly convergent subsequence. By the biorthogonality and totalness conditions of the M-basis, this subsequence must converge weakly to 0 . Hence $\varepsilon \leqslant 0$.

Recall that a norm $\|\cdot\|$ is said to have modulus of rotundity of power type $p$ if there is a $C>0$ such that for every $\varepsilon \in[0,2], \inf \{1-\|x+y\| / 2:\|x\|=\|y\|=1,\|x-y\| \geqslant$ $\varepsilon\} \geqslant C \varepsilon^{p}$. The following proof uses the fact that spaces admitting such norms are reflexive; in fact they are precisely the superreflexive spaces (see [3, Chapter IV]). 
Theorem 1.2. Suppose $p>1$ is an odd integer and suppose $X$ is nonseparable and admits a norm with modulus of rotundity of power type $p$. If every continuous symmetric $j$-linear form is weakly sequentially continuous for $j \leqslant p-1$, then $X$ does not admit a continuous p-times Gateaux differentiable bump function.

Proof: Suppose $b$ is a continuous $p$-times Gateaux differentiable bump function on $X$ such that $b(0)=1$ and $b(x)=0$ for $\|x\| \geqslant 1$. Now consider $\phi$ defined by $\phi(x)=b^{-2}(x)$ if $b(x) \neq 0$ and $\phi(x)=+\infty$ otherwise. Let $X \times \mathbb{R}$ be endowed with the norm $\|(x, r)\|=\left(\|x\|^{2}+r^{2}\right)^{1 / 2}$ where we are assuming $\|\cdot\|$ is a norm with modulus of rotundity of power type $p$ on $X$. Let $F=\{(x, r): \phi(x) \leqslant r \leqslant 4\}$. Observe that $F$ is closed. Because $X$ is reflexive, there is a point $\left(x_{0}, r_{0}\right)$ with $\left\|x_{0}\right\| \leqslant 1$ and $r_{0} \leqslant 5$ having a farthest point in $F$ (see for example, [3, Proposition II.2.7]). If $(x, r) \in F$ and $r \geqslant 3$, we have $\|x\| \leqslant 1$. Whence $\left|r-r_{0}\right| \leqslant 2$ and $\left\|x_{0}-x\right\| \leqslant 2$ and so $\left\|(x, r)-\left(x_{0}, r_{0}\right)\right\| \leqslant \sqrt{8}$. On the other hand, $(0,1) \in F$ and $\left\|\left(x_{0}, r_{0}\right)-(0,1)\right\| \geqslant$ $r_{0}-1 \geqslant 3$. Hence for farthest point $(\bar{x}, \bar{r})$, we have $\bar{r} \leqslant 3$ and $\bar{r}=\phi(\bar{x})$.

Let $\Lambda=\phi^{\prime}(\bar{x})$ and set $\psi(h)=\phi(\bar{x}+h)-\phi(\bar{x})-\Lambda(h)$. Clearly $\psi$ is $p$-times Gateaux differentiable at 0 and for $\delta>0$ chosen so that $\phi(\bar{x}+h) \leqslant 7 / 2$ for $\|h\| \leqslant \delta$, we claim

$$
\psi(h) \geqslant K\|h\|^{p} \quad \text { for some } K>0 \text { and all }\|h\| \leqslant \delta .
$$

To prove (1.1), let $\rho=\left\|\left(x_{0}, r_{0}\right)-(\bar{x}, \bar{r})\right\|$ and for $\|h\| \leqslant \delta$ consider $\eta=\|(h, \Lambda(h))\|$, then $\eta \geqslant\|h\|$. The ball of radius $\rho$ with centre $\left(x_{0}, r_{0}\right)$ is supported at $(\bar{x}, \bar{r})$ by a (unique) hyperplane which must be the graph of $\Lambda+\bar{r}$. Thus, since $\|(\cdot, \cdot)\|$ has modulus of rotundity of power type $p$, it follows from [4, Lemma 5] that

$$
\left\|\left(x_{0}, r_{0}\right)-(\bar{x}+h, \bar{r}+\Lambda(h))\right\| \geqslant C\left(\frac{\eta}{2}\right)^{p}+\rho \geqslant \frac{C}{2^{p}}\|h\|^{p}+\rho,
$$

where $C$ is the constant from the modulus of rotundity of the $\rho$-ball. By the triangle inequality, for $K=2^{-p} C$, we have

$$
\begin{aligned}
\psi(h) & =\|(\bar{x}+h, \phi(\bar{x}+h))-(\bar{x}+h, \bar{r}+\Lambda(h))\| \\
& \geqslant\left\|\left(x_{0}, r_{0}\right)-(\bar{x}+h, \bar{r}+\Lambda(h))\right\|-\left\|\left(x_{0}, r_{0}\right)-(\bar{x}+h, \phi(\bar{x}+h))\right\| \\
& \geqslant\left(K\|h\|^{p}+\rho\right)-\rho=K\|h\|^{p} .
\end{aligned}
$$

Thus (1.1) has been verified. However, $\psi$ is $p$-times Gateaux differentiable at 0 so for every $h \in S_{X}$, using the notation $h^{k}=(h, \ldots, h)$ ( $k$-times), Taylor's theorem yields:

$$
\lim _{t \rightarrow 0} t^{-p}\left[\psi(0+t h)-\psi(0)-\sum_{k=1}^{p} \frac{t^{k}}{k !} \psi^{(k)}(0)\left(h^{k}\right)\right]=0 .
$$


Since $X$ is reflexive and nonseparable, it admits an uncountable M-basis (see for example, [3, Chapter VII]). Hence by Lemma 1.1 for $h=x_{\gamma}$ some $\gamma$ we have $\psi^{(k)}(0)\left(h^{k}\right)=0$ for $k=1, \ldots, p-1$. Since $p$ is odd, by replacing $h$ with $-h$ if necessary, we also have $\psi^{(k)}(0)\left(h^{p}\right) \leqslant 0$. In particular, for $t$ sufficiently small, we have

$$
\psi(t h)=\psi(t h)-\psi(0) \leqslant \sum_{k=1}^{p} \frac{t^{k}}{k !} \psi^{(k)}(0)\left(h^{k}\right)+\frac{K}{2}\|t h\|^{p} \leqslant \frac{K}{2}\|t h\|^{p},
$$

which contradicts (1.1). Therefore $X$ cannot admit a continuous $p$-times Gateaux differentiable bump function.

The following result improves [10, Theorem 3.5].

Corollary 1.3. If $p>1$ is odd and $\Gamma$ is uncountable, then $\ell_{p}(\Gamma)$ does not admit a continuous $p$-times Gateaux differentiable bump function.

Proof: This follows from Theorem 1.2, because $\ell_{p}(\Gamma)$ admits a norm with modulus of rotundity of power type $p$ (see for example, [3, Corollary V.2.1(ii)]) and its continuous symmetric $j$-linear forms are weakly sequentially continuous for $j \leqslant p-1$ by $[\mathbf{1}$, Lemma 1$]$.

\section{UNIFORMLY DIRECTIONALLY DIFFERENTIABLE BUMP FUNCTIONS}

We shall say a function $\phi: X \rightarrow Y$ is uniformly Gateaux differentiable (UG) if for each $x \in X$ there is a continuous linear mapping $\phi^{\prime}(x): X \rightarrow Y$ such that for each $h \in S_{X}$ and $\varepsilon>0$ there exists a $\delta>0$ for which

$$
\left\|\phi(x+t h)-\phi(x)-\phi^{\prime}(x)(t h)\right\| \leqslant \varepsilon|t| \quad \text { for all }|t| \leqslant \delta, x \in X
$$

this implies $\phi$ has a uniform directional modulus of smoothness, that is, given $h \in S_{X}$ and $\varepsilon>0$, there is a $\delta>0$ such that

$$
\|\phi(x+t h)+\phi(x-t h)-2 \phi(x)\| \leqslant \varepsilon t \quad \text { for all } 0 \leqslant t \leqslant \delta, x \in X .
$$

If for each $h \in S_{X}$, there are $C_{h}$ and $\alpha_{h}>0$ such that

$$
\left\|\phi(x+t h)-\phi(x)-\phi^{\prime}(x)(t h)\right\| \leqslant C_{h}|t|^{1+\alpha_{h}} \quad \text { for all } t \in \mathbb{R}, x \in X,
$$

then we shall say $\phi^{\prime}$ is uniformly directionally Hölder. This clearly implies $\phi$ has a uniform directional modulus of smoothness of power type, that is, for each $h \in S_{X}$ there are $C_{h}>0$ and $\alpha_{h}>0$ such that

$$
\|\phi(x+t h)+\phi(x-t h)-2 \phi(x)\| \leqslant C_{h} t^{1+\alpha_{h}} \quad \text { for all } t \geqslant 0, x \in X
$$

The following observations will be useful. 
REMARK 2.1.

(a) If $f: X \rightarrow \mathbb{R}$ is Lipschitz and UG (respectively and $f^{\prime}$ is uniformly directionally Hölder) and $\phi: \mathbb{R} \rightarrow \mathbb{R}$ is Lipschitz with Lipschitz derivative, then $\phi \circ f$ is UG (respectively has uniformly directionally Hölder derivative).

(b) If $\phi: X \rightarrow Y$ is UG and $\sup \{\|\phi(x)\|: x \in X\} \leqslant N$ for some $N$, then $\phi$ is Lipschitz.

Proof: The proof of (a) is a direct computation so we now prove (b). For fixed $h \in S_{X}$ and $\varepsilon=1$ we find $\delta>0$ such that

$$
\left\|\phi(x+\delta h)-\phi(x)-\phi^{\prime}(x)(\delta h)\right\| \leqslant 1 \text { for all } x \in X .
$$

Hence $\left\|\phi^{\prime}(x)(\delta h)\right\| \leqslant 2 N+1$ and so $\left\|\phi^{\prime}(x)(h)\right\| \leqslant(2 N+1) / \delta$ for all $x \in X$. Thus letting

$$
F_{n}=\left\{h \in S_{X}:\left\|\phi^{\prime}(x)(h)\right\| \leqslant n \text { for all } x \in X\right\},
$$

we see that $\bigcup_{n=1}^{\infty} F_{n}=S_{X}$. Since each $F_{n}$ is closed, the Baire category theorem shows $\left\{h \in S_{X}:\left\|h-h_{0}\right\| \leqslant 2 r\right\} \subset F_{n_{0}}$ for some $h_{0} \in S_{X}, n_{0} \in \mathbb{N}$ and $r>0$. Letting $C=n_{0}(1+r)$ we have $\left\|\phi^{\prime}(x)(h)\right\| \leqslant C$ for all $x \in X$ whenever $\left\|h-h_{0}\right\| \leqslant r(h$ need not belong to the sphere). Now for any $x \in X$ and any $h \in S_{X}$ we have

$$
\left\|\phi^{\prime}(x)(r h)\right\|=\left\|\phi^{\prime}(x)\left(-h_{0}\right)+\phi^{\prime}(x)\left(h_{0}+r h\right)\right\| \leqslant 2 C .
$$

Hence $\sup \left\{\left\|\phi^{\prime}(x)\right\|: x \in X\right\} \leqslant 2 C / r$ which means that $\phi^{\prime}$ is bounded; thus $\phi$ is Lipschitz.

Observe that the proof of (b) easily adapts to show that a possibly unbounded uniformly continuous UG function is globally Lipschitz. However, we shall not need to use this fact in the sequel. The next lemma will enable us to build nontrivial smooth convex functions from certain smooth bump functions; this is an alternative approach to the Leduc type function used in the proof of [3, Theorem V.3.2].

LEMma 2.2. Suppose $b: X \rightarrow[0,1]$ is a bump function such that $b(x)=1$ if $\|x\| \leqslant 1 / r$ and $b(x)=0$ if $\|x\| \geqslant 1 / 2$ where $r>2$. Let $\phi(x)=\sum_{n=1}^{\infty} r^{n}\left[1-b\left(r^{-n} x\right)\right]$. Then

$$
\phi(0)=0 \quad \text { and } \quad \max \left\{0, \frac{1}{r}(\|x\|-r)\right\} \leqslant \phi(x) \leqslant r^{2}(\|x\|+1) \quad \text { for all } x \in X ;
$$

(a) if b has uniform directional modulus of smoothness of power type, so does $\phi$;

(b) if b has uniform directional modulus of smoothness, so does $\phi$. 
Proof: Clearly $\phi(0)=0$ so we check the other parts of $(2.1)$. If $\widetilde{b}(x)=1-b(x)$, then $\widetilde{b}\left(r^{-(n+1)} x\right)=0$ for $r^{n} \geqslant\|x\|$. Thus for $\|x\| \leqslant 1,(2.1)$ holds. If $r^{n-1} \leqslant\|x\| \leqslant r^{n}$, then

$$
\phi(x)=\sum_{k=1}^{n} r^{k} \widetilde{b}\left(r^{-k} x\right) \leqslant \sum_{k=1}^{n} r^{k}<r^{2} \cdot r^{n-1} \leqslant r^{2}\|x\| .
$$

For the other inequality, notice that if $r^{n} \leqslant\|x\| \leqslant r^{n+1}$, we have

$$
\phi(x) \geqslant r^{n} \tilde{b}\left(r^{-n} x\right)=r^{n} \geqslant \frac{1}{r}\|x\| .
$$

Since $\phi(x) \geqslant 0$ for all $x$ it is clear that (2.1) holds.

To prove (a), let $\widetilde{b}(x)=1-b(x)$ and observe that

$$
\begin{aligned}
|\phi(x+t h)+\phi(x-t h)-2 \phi(x)| & =\left|\sum_{n=1}^{\infty} r^{n}\left[\widetilde{b}\left(r^{-n}(x+t h)\right)+\widetilde{b}\left(r^{-n}(x+t h)\right)-2 \widetilde{b}\left(r^{-n} x\right)\right]\right| \\
& \leqslant \sum_{n=1}^{\infty} r^{n}\left[C_{h}\left(r^{-n} t\right)^{1+\alpha_{h}}\right] \\
& =t^{1+\alpha_{h}} C_{h} \sum_{n=1}^{\infty}\left(r^{\alpha_{h}}\right)^{-n}=K_{h} t^{1+\alpha_{h}} .
\end{aligned}
$$

For (b), observe that for any $x_{0} \in X$, there is at most one $f_{n}(x)=r^{n}\left(1-b\left(r^{-n} x\right)\right)$ which is nonconstant on $\left\{x:\left\|x-x_{0}\right\| \leqslant 1\right\}$. Thus the modulus of smoothness depends only on one such $f_{n}$ which has the same modulus as $b$ (this proof also works for (a)).

Notice that Remark 2.1(b) implies that if a Lipschitz function $\phi$ has a uniformly Gateaux second derivative, then $\phi^{\prime}$ is Lipschitz. In particular, if $\phi$ is a bump function, the space must be superreflexive [3, Theorem V.3.2]. We now sharpen this result.

THEOREM 2.3. If $b$ is a bounded bump function on $X$ such that $b^{\prime}$ satisfies a uniform directional power type condition, then $X$ admits a norm $\nu$ with Hölder derivative on its sphere (that is, there exist $K>0,0<\alpha \leqslant 1$ such that $\left\|\nu^{\prime}(x)-\nu^{\prime}(y)\right\| \leqslant K\|x-y\|^{\alpha}$ for all $x, y$ with $\left.\nu(x)=\nu(y)=1\right)$.

Proof: (a) According to Remark 2.1, by composing $b$ with an appropriate realvalued function, we may assume $b$ satisfies the hypothesis of Lemma 2.2 . Let $\phi$ be the function given by the Lemma 2.2. Following [3, Theorem V.3.2], let $\psi$ be defined by

$$
\psi(x)=\inf \left\{\sum_{i=1}^{n} \alpha_{i} \phi\left(x_{i}\right): x=\sum_{i=1}^{n} \alpha_{i} x_{i}, \alpha_{i} \geqslant 0, \sum_{i=1}^{n} \alpha_{i}=1, n \in \mathbb{N}\right\}
$$

Then $\psi$ is convex and satisfies (2.1). Thus the proof of [9, Proposition 1.6] shows $\psi$ is Lipschitz. Now, for $h \in S_{X}$ and $t \geqslant 0$ fixed and any $x \in X$, we choose a convex 
combination $x=\sum_{i=1}^{n} \alpha_{i} x_{i}$ satisfying $\sum_{i=1}^{n} \alpha_{i} \phi_{i}\left(x_{i}\right)<\psi(x)+\varepsilon t^{1+\alpha_{h}}$. Hence,

$$
\begin{aligned}
\psi(x+t h) & +\psi(x-t h)-2 \psi(x) \leqslant \psi(x+t h)+\psi(x-t h)-2 \sum_{i=1}^{n} \alpha_{i} \phi\left(x_{i}\right)+2 \varepsilon t^{1+\alpha_{h}} \\
& \leqslant \sum_{i=1}^{n} \alpha_{i} \phi\left(x_{i}+t h\right)+\sum_{i=1}^{n} \alpha_{i} \phi\left(x_{i}-t h\right)-2 \sum_{i=1}^{n} \alpha_{i} \phi\left(x_{i}\right)+2 \varepsilon t^{1+\alpha_{h}} \\
& =\sum_{i=1}^{n} \alpha_{i}\left[\phi\left(x_{i}+t h\right)+\phi\left(x_{i}-t h\right)-2 \phi(x)\right]+2 \varepsilon t^{1+\alpha_{h}} \\
& \leqslant\left(K_{h}+2 \varepsilon\right) t^{1+\alpha_{h}}
\end{aligned}
$$

Motivated by [2, Proposition 2.2], we consider the following sets:

$$
F_{n}=\left\{h \in S_{X}: \psi(x+t h)+\psi(x-t h)-2 \psi(x) \leqslant n t^{1+\frac{1}{n}} \quad \text { for all } t \geqslant 0, x \in X\right\} .
$$

Because $\psi$ is Lipschitz, we know $\psi(x+h)+\psi(x-h)-2 \psi(x) \leqslant n t^{1+1 / n}$ for all $t \geqslant 1$, $h \in S_{X}, x \in X$ and $n \geqslant 2 L$ where $L$ is the Lipschitz constant of $\psi$. This with (2.2), shows $\bigcup_{n=1}^{\infty} F_{n}=S_{X}$. The continuity of $\psi$ ensures each $F_{n}$ is closed, so the Baire category theorem shows there are $h_{0} \in S_{X}, n_{0} \in \mathbb{N}$ and $\delta>0$ such that $\left\{h \in S_{X}:\left\|h-h_{0}\right\| \leqslant 4 \delta\right\} \subset F_{n_{0}}$. We let $\alpha=1 / n_{0}$ and $C=n_{0}(1+2 \delta)^{1+\alpha}$ to obtain (for $h$ not necessarily in $S_{X}$ )

(2.3) $\psi(x+t h)+\psi(x-t h)-2 \psi(x) \leqslant C t^{1+\alpha} \quad$ for all $t \geqslant 0, x \in X,\left\|h-h_{0}\right\| \leqslant 2 \delta$.

If $h \in S_{X}$ is arbitrary, then we can write $\delta h=\left(-h_{0}\right) / 2+\left(h_{0}+2 \delta h\right) / 2$. Thus using (2.3) and the convexity of $\psi$, for $K=C / \delta^{1+\alpha}$, we obtain

$$
\psi(x+t h)+\psi(x-t h)-2 \psi(x) \leqslant K t^{1+\alpha} \quad \text { for all } t>0, x \in X \text { and } h \in S_{X} .
$$

Hence [3, Lemma V.3.5] shows that $\psi^{\prime}$ is $\alpha$-Hölder on $X$. Now set $\widetilde{\psi}(x)=$ $(\psi(x)+\psi(-x)) / 2$. Then $\widetilde{\psi}^{\prime}$ is $\alpha$-Hölder and Lemma 2.2 shows

$$
\widetilde{\psi}(0)=0 \quad \text { and } \quad \max \left\{0, \frac{1}{r}(\|x\|-r)\right\} \leqslant \widetilde{\psi}(x) \leqslant \phi(x) \leqslant r^{2}(\|x\|+1) .
$$

The convexity and symmetry of $\widetilde{\psi}$ along with (2.4), show that the set $\{x: \widetilde{\psi}(x) \leqslant 2\}$ is bounded, convex, symmetric and has nonempty interior. Therefore, one can use the implicit function theorem as in the proof of [3, Theorem V.3.2] to show the norm whose unit ball is $\{x: \widetilde{\psi}(x) \leqslant 2\}$ has $\alpha$-Hölder derivative on its sphere. 
In the above theorem, if $\alpha_{h} \geqslant \alpha$ for each $h \in S_{X}$, then the proof shows that $X$ admits a norm with $\alpha$-Hölder derivative on its sphere. We now look at some properties of spaces that admit UG bump functions. In what follows, $f \square g(x)=\inf \{f(y)+g(x-y)$ : $y \in X\}$ denotes the infimal convolution of $f$ and $g$; the subdifferential of $f$ at $x$ is defined and denoted by $\partial f(x)=\left\{\Lambda \in X^{*}: \Lambda(y-x) \leqslant f(y)-f(x)\right.$ for all $\left.y \in X\right\}$.

LEMMa 2.4. Suppose the function $g$ is a continuous convex $U G$ function. If $f$ is convex, then $f \square g$ is continuous convex and $U G$ provided it is finite valued.

Proof: Let $h \in S_{X}$ be fixed, let $\varepsilon>0$ and choose $\delta>0$ such that

$$
g(x+t h)+g(x-t h)-2 g(x) \leqslant \frac{\varepsilon}{2} t \text { for all } 0 \leqslant t<\delta, x \in X .
$$

Let $r$ denote the function $f \square g$. It is well-known that $r$ is convex, and continuous provided it is finite valued. Fix $x \in X$ and $0<t \leqslant \delta$. Now choose $y \in X$ such that $r(x)+(\varepsilon / 4) t>g(y)+f(x-y)$. From this and (2.5) we have

$$
\begin{aligned}
r(x+t h)+r(x-t h)-2 r(x) \leqslant & r(x+t h)+r(x-t h)-2[g(y)+f(x-y)]+\frac{\varepsilon}{2} t \\
\leqslant & g(y+t h)+f(x-y)+g(y-t h)+f(x-y) \\
& -2[g(y)+f(x-y)]+\frac{\varepsilon}{2} t \\
= & g(y+t h)+g(y-t h)-2 g(y)+\frac{\varepsilon}{2} t \leqslant \varepsilon t .
\end{aligned}
$$

Since $r$ is continuous and convex, this shows $r$ is UG. Indeed if $\Lambda \in \partial r(x)$, then

$0 \leqslant r(x+t h)-r(x)-\Lambda(t h) \leqslant r(x+t h)-r(x)+r(x-t h)-r(x) \leqslant \varepsilon|t| \quad$ for $\quad|t| \leqslant \delta$.

Recall that a norm is strictly convex if its sphere contains no line segments. It is easy to show that a norm is Gateaux differentiable if its dual norm is strictly convex (see [3, Proposition II.1.6(i)]), whereas the converse can fail badly (see [3, Theorem VII.5.2 and Theorem VII.5.4]). The next theorem is in contrast to Haydon's results [6, 7] showing there are spaces admitting Lipschitz $C^{\infty}$-smooth bump functions on a space that has no Gateaux smooth norm.

Theorem 2.5. Suppose $X$ admits a bounded UG bump function. Then the following hold.

(a) Every convex function $f$ bounded on bounded sets can be approximated uniformly on bounded sets by $U G$ convex functions.

(b) $X$ admits a norm whose dual is strictly convex. 
Proof: (a) We construct a continuous convex $\psi$ as the proof of Theorem 2.3. Arguing as in (2.2), for fixed $h \in S_{X}$ and $\varepsilon>0$, there is a $\delta>0$ such that

$$
\psi(x+t h)+\psi(x-t h)-2 \psi(x) \leqslant \varepsilon t \quad \text { for all } 0<t<\delta, x \in X
$$

As in Lemma 2.4, this inequality means $\psi$ is UG. Moreover, as in (2.4) in the proof of Theorem 2.3, $\psi(0)=0$ and $\psi(x) \geqslant \max \{0,\|x\| / r-1\}$ for some $r>0$. Let $\psi_{n}(x)=$ $\psi\left(2 n^{2} r x\right)$. Then $\psi_{n}$ is $\mathrm{UG}, \psi_{n}(0)=0$, and one easily checks $\psi_{n}(x) \geqslant n\|x\|-1 / n$ for all $x$. For $n>\|\Lambda\|$ where $\Lambda \in \partial f(0), \psi_{n} \square f$ is finite valued and hence continuous convex and UG by Lemma 2.4. From [8, Lemma 2.4] it follows that $\psi_{n}$ a $f$ converges to $f$ uniformly on bounded sets.

(b) Using (a) one can easily construct a UG convex function $f$ such that $f(0)=0$ and $B=\{x: f(x) \leqslant 1\}$ is a bounded symmetric convex set with nonempty interior. Let $\|\cdot\|$ denote the norm whose unit ball is $B$. If $\|\cdot\|$ is not Gateaux differentiable, then it would not be Gateaux differentiable on some finite dimensional subspace and hence it would not be Fréchet differentiable on this subspace; this would contradict the implicit function theorem. Thus we have $\|\cdot\|^{\prime}(x)=f^{\prime}(x) /\left(f^{\prime}(x)(x)\right)$. Now suppose $\phi, \psi \in X^{*}$ are such that $\|\phi\|=\|\psi\|=1$ and $\|\phi+\psi\|=2$. To prove strict convexity, we show $\phi=\psi$. Choose $x_{n}$ such that $\left\|x_{n}\right\|=1$ and $(\phi+\psi)\left(x_{n}\right) \rightarrow 2$. Hence $\phi\left(x_{n}\right) \geqslant 1-\varepsilon_{n}$ and $\psi\left(x_{n}\right) \geqslant 1-\varepsilon_{n}$ where $\varepsilon_{n} \downarrow 0$. By the Brøndsted-Rockafellar theorem [9, Theorem 3.18], there are $\phi_{n}, \psi_{n} \in X^{*}$ such that $\phi_{n}$ and $\psi_{n}$ attain there norm on $B$ at $u_{n}, v_{n}$ where $\left\|u_{n}-x_{n}\right\| \leqslant \sqrt{\varepsilon_{n}},\left\|v_{n}-x_{n}\right\| \leqslant \sqrt{\varepsilon_{n}},\left\|\phi_{n}-\phi\right\| \leqslant \sqrt{\varepsilon_{n}}$ and $\left\|\psi_{n}-\psi\right\| \leqslant \sqrt{\varepsilon_{n}}$. Now for some $a_{n}, b_{n}$ we have $a_{n} \phi_{n}=f^{\prime}\left(u_{n} /\left\|u_{n}\right\|\right)$ and $b_{n} \psi_{n}=f^{\prime}\left(v_{n} /\left\|v_{n}\right\|\right)$. Since $f$ is $U G$ and $\left\|u_{n}-v_{n}\right\| \rightarrow 0$, this implies $a_{n} \phi_{n}-b_{n} \psi_{n} \stackrel{w^{*}}{\rightarrow} 0$. By passing to a subsequence, we may assume $a_{n^{\prime}} \rightarrow \bar{a}$ and $b_{n^{\prime}} \rightarrow \bar{b}$, and so $\bar{a} \phi_{n^{\prime}}-\bar{b} \psi_{n^{\prime}} \stackrel{w^{*}}{\rightarrow} 0$. Moreover, $\bar{a} \phi_{n}-\bar{b} \psi_{n} \rightarrow \bar{a} \phi-\bar{b} \psi$. Hence $\bar{a} \phi=\bar{b} \psi$ which means $\bar{a}=\bar{b}$ because $\|\phi\|=\|\psi\|$ and $\|\phi+\psi\|=2$. Since $\inf \left\{\left\|f^{\prime}(x)\right\|:\|x\|=1\right\}>0, \bar{a} \neq 0$ and $\phi=\psi$ as desired.

We do not know if the norm given in (b) is UG. Moreover, it is not clear if there is an analog of (a) for nonconvex continuous functions. However, we have the following partial redress. First recall that a norm is said to be locally uniformly rotund (LUR) if $\left\|x_{n}-x\right\| \rightarrow 0$ whenever $x, x_{n} \in B_{X}$ are such that $\left\|x_{n}+x\right\| \rightarrow 2$; see [3, Chapter VII] for a thorough account on spaces admitting LUR norms.

Corollary 2.6. Suppose $X$ admits a bounded UG bump function and a LUR norm. If $f: X \rightarrow \mathbb{R}$ is continuous and $\varepsilon>0$, then there is a continuous Gateaux differentiable function $g$ such that $|g(x)-f(x)|<\varepsilon$ for all $x \in X$.

Proof: By Theorem 2.5(b) and Asplund's averaging theorem [3, Theorem II.4.1], $X$ has a LUR with strictly convex dual norm so we can apply [3, Theorem VIII.3.12(ii)]. 


\section{REFERENCES}

[1] R. Bonic and J. Frampton, 'Smooth functions on Banach manifolds', J. Math. Mech. 15 (1966), 877-899.

[2] J.M. Borwein and D. Noll, 'Second order differentiability of convex functions in Banach spaces', Trans. Amer. Math. Soc. 342 (1994), 43-81.

[3] R. Deville, G. Godefroy and V. Zizler, Smoothness and renormings in Banach spaces, Monographs and Surveys in Pure and Applied Mathematics 64 (Pitman, 1993).

[4] M. Fabian, D. Preiss, J.H.M. Whitfield and V. Zizler, 'Separating polynomials on Banach spaces', Quart. J. Math. Oxford Ser. (2) 40 (1989), 409-422.

[5] M. Fabian, J.H.M. Whitfield and V. Zizler, 'Norms with locally Lipschitzian derivatives', Israel J. Math. 44 (1983), 262-276.

[6] R. Haydon, 'A counterexample to several questions about scattered compact spaces', Bull. London Math. Soc. 22 (1990), 261-268.

[7] R. Haydon, Normes et partitions de l'unité indéfiniment différentiables sur certaines espaces de Banach, C. R. Acad. Sci. Paris Sér. I 315 (1992), 1175-1178.

[8] D. McLaughlin, R. Poliquin, J. Vanderwerff and V. Zizler, 'Second-order Gâteaux differentiable bump functions and approximations in Banach spaces', Canad. J. Math. 45 (1993), 612-625.

[9] R.R. Phelps, Convex functions, monotone operators and differentiability, Lecture Notes in Mathematics 1364 (Springer-Verlag, Berlin, Heidelberg, New York, 1989).

[10] S. Troyanski, 'Gâteaux differentiable norms in $L_{p}$ ', Math. Ann. 287 (1990), 221-227.

Department of Mathematics

Kwantlen College

Surrey BC

Canada V3T 5 H8
Department of Mathematics and Statistics

Simon Fraser University

Burnaby BC

Canada V5A 156 